\title{
Costo de capital bajo riesgos asimétricos en el mercado de valores mexicano
}

\author{
M. Rodríguez, ${ }^{8}$ K. Cortez $z^{9}$ у H. García ${ }^{10}$
}

\section{Resumen}

El siguiente artículo tiene como fin calcular el costo de financiamiento de las empresas al momento de captar recursos de la Bolsa Mexicana de Valores, costo al que también se denomina costo de capital o tasa de rendimiento mínimo aceptable. Utilizamos la metodología del capital asset price model (CAPM) propuesta por W. Sharpe (1964) y contrastamos este análisis con la metodología formulada y adaptada por J. Estrada (2000 y 2001) llamada capital asset price model modificated (CAPM-M). Para ello, realizamos un estudio basado en 35 empresas que componen el índice de precios y cotizaciones de la Bolsa Mexicana de Valores durante el periodo de 2000 a 2007. Estudios como los de J. Estrada (2000 y 2001) han encontrado que en una economía emergente como la de México el CAPM arroja resultados menos reales que el CAPM-M debido a que este último método elimina la hipótesis de normalidad. En el estudio encontramos que la $R^{2}$ ajustada en el CAPM-M es mayor que en el CAPM tradicional y que las betas son menores en el CAPM que en el CAPM-M. En ambos modelos son consistentes, sin embargo, el modelo más eficiente es el CAPM-M.

Números de clasificación: JEL:

Palabras clave: costo de capital, riesgo a la baja, índice de precios y cotizaciones.

\section{Abstract}

This paper analyzed the cost of capital in the Mexican stock market. The study is based on the traditional capital asset price model (CAPM), Sharpe (1964) and also use more contextual methodology application for emerging economies CAPM-modified by Estrada (2000 y 2001) previous studies conclude due to the typical bias in a emerging stock price market that the modified version of the CAPM shows strongest results that the orthodox methodology, the bias are concentrated in the normal statistical data. Our recent study includes the years from 2000 to 2007 for 35 most representative Mexican public companies traded in the Mexican Stock Market. In the study we found that the $R^{2}$ ajustada in the CAPM-M is greater than in the traditional and the betas are smaller in the CAPM than in the CAPM-M. CAPM and that both models are consistent, nevertheless the model most efficient is the CAPM-M.

\section{Numbers of classification: JEL:}

Key words: Equity cost, Down risk, Shares price.

8 Investigadora y catedrática del posgrado de FACPYA de la Universidad Autónoma de Nuevo León. [marthadelpilar2000@yahoo.com].

9 Consultor de organismos públicos y privados con doctorado en administración de empresas por la Universidad de Barcelona. [kcortez@facpya.uanl.mx].

10 Profesor de finanzas en la Texas A\&M International University [hgarcianunez@hotmail.com]. 


\section{INTRODUCCIÓN}

Es importante que los involucrados en la toma de decisiones financieras conozcan métodos que les permitan la estimación aproximada de los costos de financiamiento, para tomar decisiones que maximicen la creación de valor de los inversionistas.

Existe un gran número de aplicaciones financieras que necesitan del cálculo de los costos de financiamiento, por ejemplo: $i$ ) la estimación de las métricas de desempeño, como el valor económico agregado conocido por sus siglas en inglés como EVA (economic value added); ii) la evaluación de proyectos de inversión, ya que mediante la obtención de la tasa de descuento (costo de capital) podemos transferir los flujos de efectivo que generará la empresa ante la presencia de nueva inversión, y finalmente iii) la información para la toma de decisiones de los inversionistas, ya que se pueden realizar comparaciones entre los costos de financiamiento entre sectores o entre empresas.

En este artículo sólo nos centraremos en el cálculo de la tasa mínima requerida por el accionista, ${ }^{8}$ llamada habitualmente "costo de capital", que comprende el financiamiento interno, el cual considera los recursos financieros que la empresa genera para sí misma (utilidades retenidas, capital fundacional, ampliaciones de capital, amortizaciones, provisiones). La muestra del estudio comprende a las empresas mexicanas más representativas de la economía, esto es, las empresas que componen el índice de precios y cotizaciones (IPC), tomando como referencia el periodo de 2000 a 2007.

Existen diferentes maneras de medir el costo de capital, como el capital asset price model (CAPM) y arbitrage price theory (APT). El modelo que emplearemos es el CAPM desarrollado por W. Sharpe (1964) y modificado por J. Estrada (2000 y 2001). A continuación probaremos que en una economía emergente como la de México, el CAPM modificado es mejor que el CAPM desarrollado por W. Sharpe (1964). Para ello dividimos esta hipótesis en dos:

1) Las betas calculadas a partir del CAPM tradicional son más pequeñas que las obtenidas mediante la metodología del CAPM-M en las empresas que constituyen el índice de precios y cotizaciones (IPC).

2) La utilización del CAPM-M para calcular la beta modificada aumenta la emulación del modelo mediante un incremento de la $R^{2}$ ajustada en promedio en las empresas que constituyen el IPC en México.

\footnotetext{
${ }^{8}$ El costo de los capitales propios se define como la tasa mínima que debe obtenerse en la colocación de medios financieros para que se mantenga la riqueza de los accionistas.
} 


\section{ANTECEDENTES}

Uno de los más importantes supuestos en la teoría financiera sobre la valoración de un activo financiero es el supuesto de competencia perfecta. Un mercado perfecto surge bajo tres condiciones:

1) Cuando el número de activos financieros es igual al número de estados de la naturaleza.

2) Cuando existe una distribución de probabilidad única con la cual valorar los activos financieros.

3) La introducción de una nueva acción dentro del mercado de capitales puede ser valorada de una forma única.

En el modelo clásico del CAPM los inversionistas maximizan una función de utilidad considerando la media y la varianza.

La estructura estándar del modelo de varianza media de un inversionista muestra una función de utilidad determinada en su totalidad por la media $\left(\mu_{p}\right)$ y la varianza $\left(\sigma_{i}^{2}\right)$ de los rendimientos del portafolio de un inversor; esto es, $U=U\left(\mu_{p}, \sigma^{2}\right)$. En tal estructura, el riesgo de un activo $i$ tomado individualmente es medido por la desviación estándar de sus rendimientos $\left(\sigma_{i}\right)$, el cual está dado por:

$$
\sigma_{i}=\sqrt{\left.E\left(R_{i}-\mu\right)^{2}\right)}
$$

El modelo CAPM se podría traducir como el "modelo de equilibrio de los activos financieros". Es utilizado para calcular el costo de capital de las empresas públicas. Las hipótesis según las cuales se fundamenta son las siguientes:

a) La cartera de los inversionistas se encuentra diversificada y, por tanto, únicamente el riesgo sistemático es relevante. Es el riesgo que afecta prácticamente a todo el mercado, ya que es originado por factores políticos, macroeconómicos y sociales que modifican significativamente la percepción de las expectativas del inversionista sobre las utilidades de las empresas.

b) Los rendimientos de las acciones siguen una distribución normal, lo que entre otras cosas significa que dichos rendimientos son simétricos respecto a su media.

c) Los movimientos específicos de un título tienen efectos relativamente 
pequeños en el total del mercado. Los factores que influyen al rendimiento de los accionistas $\left(R_{i, t}\right)$ son el riesgo de la inversión, las tasas de interés y la experiencia de los administradores.

El CAPM capta dos de los primeros conceptos; la siguiente expresión determina el rendimiento esperado:

$$
E\left(R_{j, t}\right)=R_{f t}+\beta\left[E\left(R_{m, t}\right)-R_{f t}\right]
$$

donde: $E\left(R_{j, t}\right)=$ rentabilidad esperada de la acción $j$ en el periodo $t$.

$R_{j, t}=$ tasa libre de riesgo que se mantiene en el tiempo $t$.

$\beta_{\mathrm{j}}=$ medida del riesgo sistemático de la empresa $j$.

$E\left(R_{m, t}\right)=$ rentabilidad esperada del mercado en el periodo $t$.

$\left[E\left(R_{m, t}\right)-R_{f t}\right]=$ ganancia esperada por riesgo del mercado.

Todas las variables "esperadas" representan las expectativas de los inversionistas, por lo cual es difícil hacer pruebas empíricas exactas, ya que usualmente se toman los valores promedio de los datos históricos.

Existe una gran cantidad de estudios que tratan de criticar los pronósticos del CAPM. El trabajo que más efecto ha tenido es el desarrollado por Fama y French (1992), cuyos autores demostraron que durante el periodo de 1963 a 1990 la relación de las rentabilidades de las acciones con sus betas fue muy pequeña. Esta misma conclusión la obtuvo Harvey (1995).

Para solucionar esta situación, Godfrey y Espinosa (1996) propusieron sumar a la tasa libre de riesgo el diferencial entre el rendimiento de un bono del Estado denominado en dólares y el rendimiento de un bono comparable de Estados Unidos; el problema de este cálculo es que no todas la tasas libres de riesgo están denominadas en dólares. Otra opción se basó en la calificación de crédito. Por ejemplo, Erb et al. (1996) reportaron que estas calificaciones se encontraban significativamente relacionadas con los rendimientos de las acciones. El problema con esta metodología es que sólo se puede utilizar en el nivel país y no en el de la empresa. Existe otra vía que trata de mejorar al CAPM tradicional, de la cual hablaremos a continuación. 


\section{RIESGO A LA BAJA: EL CAPM MODIFICADO}

Las hipótesis del CAPM no son aplicables al caso mexicano debido a que los rendimientos de las acciones son asimétricos respecto a su media. Además, el alta concentración de este mercado ocasiona que los movimientos específicos de una acción afecten al total de éste. Asimismo, Estrada (2001, p. 63) menciona que en los países emergentes se suelen obtener costos de capital bastante bajos y poco intuitivos respecto al riesgo que representa el invertir en ellos. En un principio se pensaba que esto se debía a que los mercados emergentes tenían una baja correlación respecto a las principales bolsas del mundo, es decir, estaban "aislados". Sin embargo, ahora se ha observado que esto es muy relativo, ya que en condiciones normales la correlación entre los países desarrollados y los emergentes es baja; pero cuando las bolsas de los países desarrollados caen, éstas arrastran a todas las bolsas del mundo y, por lo tanto, la correlación es alta. Para tratar esta problemática, J. Estrada (2000 y 2001) propone un cambio metodológico al CAPM con el fin de aplicarlo a los datos de los países emergentes. A esta nueva versión le llamaremos CAPM modificado (CAPM- $M$ ). EL CAPM- $M$ tiene sus orígenes en el riesgo a la baja. Markowitz (1959) señalaba que para generar portafolios eficientes había que considerar la variabilidad negativa de los rendimientos, ya que la preocupación de los inversionistas se centra en esta cuestión. Bawa y Lindenberg (1977) desarrollaron un modelo de riesgo a la baja y demostraron que se comportaba como el CAPM tradicional. Posteriormente, Harlow y Rao (1989) redefinieron el modelo anterior, obteniendo buenos resultados. Más tarde, Sortino y Van der Meer (1991) mostraron que la media de la varianza a la baja funcionaba mejor que la media de la varianza. Estrada (2000 y 2001) consideró este riesgo no deseado y desarrolló el $C A P M-M$.

J. Estrada (2002) propone otra estructura al modelo clásico del CAPM, en la que los inversionistas maximizan una función de utilidad considerando la media y la varianza. Sin embargo, argumenta que la semivarianza de los rendimientos puede ser usada para generar una hipótesis de comportamiento adicional, esto es, modelo de comportamiento media, semivarianza. La función de utilidad queda expresada como: $U=U\left(\mu_{p}, \sum_{p}^{2}\right)$ determinada $\left(\mu_{p}\right)$ y la $\left(\sum_{p}^{2}\right)$, que denota la varianza hacia descendente de los rendimientos del portafolio de un inversor. En tal estructura, el riesgo de un activo $i$ tomado individualmente es medido por la semidesviación a la baja de los rendimientos, dada por:

$$
S_{i}=\sqrt{E\left\{\operatorname{Min}\left[\left(R_{i}-\mu_{i}\right), 0\right]^{2}\right\}}
$$


Los supuestos del modelo son muy similares a los del CAPM tradicional, sin embargo, no requiere simetría en la distribución de rendimientos. Para determinar el costo de capital a través del $M-C A P M$ se utiliza la siguiente expresión:

$$
E\left(R_{j, t}\right)=R_{f t}+\beta_{d}\left[E\left(R_{m, t}\right)-R_{f, t}\right]
$$

Casi todas las variables de la ecuación 2 correspondientes al CAPM tradicional se mantienen, con excepción de la $B_{d}$, llamada d-beta. El parámetro $\beta d$ indica la sensibilidad del rendimiento de cada acción $R_{i, t}$ respecto al rendimiento del mercado $R_{m, t}$, cuando ambas caen simultáneamente. El cálculo de la $\beta d$ se puede llevar a cabo de dos formas:

a) Realizando una regresión como sigue:

$$
\operatorname{Min}\left(R_{j, t}-R_{j, p} ; 0\right)=\alpha+\beta_{d} \operatorname{Min}\left(R_{m, t}-R_{j, p} ; 0\right)
$$

donde: $\quad R_{i, t}=$ rentabilidad de la acción $j$ en el periodo $t$.

$R_{j p}=$ promedio histórico de la rentabilidad de la acción $j$.

$R_{m t}=$ rentabilidad del mercado en el periodo $t$.

$R_{m p}=$ promedio histórico de la rentabilidad del mercado.

b) Calculando la siguiente fórmula:

$$
\begin{gathered}
\beta_{d}=\frac{\operatorname{SCov}\left(R_{j, t}, R_{m t}\right)}{S \sigma_{R m t}^{2}} \\
\operatorname{SCov}\left(R_{j, t}, R_{m t}\right)=\left(\frac{1}{n}\right) \sum_{i=1}^{n}\left[\operatorname{Min}\left(R_{j, t}-R_{j p} ; 0\right) \cdot \operatorname{Min}\left(R_{m t}-R_{m p} ; 0\right)\right] \\
S \sigma_{R m t}^{2}=\left(\frac{1}{n}\right) \sum_{i=1}^{n}\left[\operatorname{Min}\left(R_{m t}-R_{m p} ; 0\right)\right]^{2}
\end{gathered}
$$

$\operatorname{SCov}\left(R_{j, t}, R_{m t}\right)=$ semicovarianza entre los rendimientos de la acción $j$ $S \sigma_{R m t}^{2}=$ semivarianza del mercado. $n=$ número de observaciones. 


\section{Muestra de empresas}

La muestra que usamos corresponde a las empresas que forman parte del IPc de la Bolsa Mexicana de Valores (BMv) del año 2007. Empleamos el total de empresas que conforman el IPc, esto es, 35 compañías mexicanas durante el periodo 2000-2007, usando datos mensuales. Esta muestra incluye las compañías más representativas, asimismo, jerarquizamos la muestra por sectores para analizar el costo de capital por sector.

\section{Selección de variables}

Para calcular el costo de capital es necesario estimar tres variables: la tasa libre de riesgo $\left(R_{f t}\right)$, el rendimiento de mercado $\left(R_{m t}\right)$ y el rendimiento del precio de las acciones de cada compañía $\left(R_{j, t}\right)$. En nuestro análisis utilizaremos los Certificados de la Tesorería del Estado (Cetes) a un plazo de 28 días, como tasa libre de riesgo. ${ }^{8}$ Por otra parte, se tomará como rendimiento de mercado $\left(R_{m t}\right)$ las diferencias logarítmicas (mensuales) del índice de precios y cotizaciones. ${ }^{9}$ Asimismo, se calcularán los las diferencias logarítmicas (mensuales) en los precios de las acciones $\left(R_{j, t}\right)$ para las compañías que seleccionamos. ${ }^{10}$ L. Bachelier (1900) planteó la idea de que los incrementos en los precios son copia de una ley normal centrada y con varianza constante. Sin embargo, Osborne (1959) propuso que los incrementos de los logaritmos de los precios son independientes y están idénticamente distribuidos. Por ello, realizamos la transformación expuesta por Osborne (1959).

Empleamos el contraste de Dickey-Fuller aumentado (DFA), como se puede observar en el anexo 1 ; los valores del estadístico $t$ generalmente caen dentro del área de rechazo de la $H_{0}$, por lo que no encontramos existencia de raíces unitarias, lo que nos indica que las series son estacionarias respecto a su media, aunque para el caso de la acción de Famsa, oma y Pinfra se acepta $H_{0}$ debido a que son acciones con pocas observaciones y no se trató de ajustar la serie a primeras diferencias debido al reducido número de observaciones.

Para obtener el CAPM se necesitan: la beta $\left(\beta_{j}\right)$ de cada empresa y la prima de mercado. A continuación realizaremos los cálculos para estos parámetros $\mathrm{y}$,

\footnotetext{
${ }^{2}$ En ocasiones la tasa de Cetes no supera la inflación medida a través del cambio porcentual del índice nacional de precios al consumidor (INPC); por tanto, cuando esto sucede se toma el INPC como tasa libre de riesgo. Sin embargo, en nuestro caso esto no se aplica.

${ }^{3}$ Equivale al IBEX de España o al S\&P500 de Estados Unidos.

${ }^{4}$ Las tasas de Cetes se obtuvieron del Banco de México <http://www.banxico.org.mx>. La información del mercado accionario en Invertia Plus $<$ http://mx.plus.invertia.com $>$.
} 
por último, haremos unas modificaciones para determinar el costo de capital a partir de la metodología del $M-C A P M$.

\section{Cálculo del costo de capital utilizando el CaPM $\left(\beta_{J}\right)$}

\section{Beta (ß)bajo el enfoque del CAPM}

La beta mide la sensibilidad de la rentabilidad de la acción en relación con la del mercado. Si una beta es superior a la unidad, entonces es muy sensible a los cambios que se presentan en éste, mientras que una beta inferior a la unidad indica lo contrario. El signo de la beta señala el sentido del movimiento de la acción en relación con el mercado. Fernández (2001, p. 399) menciona que en España las betas suelen tomar valores de 0.7 a 1.3 en $80 \%$ de los casos.

Para calcular la beta utilizaremos los rendimientos mensuales de los precios de cada una de las acciones, ${ }^{8}$ así como los rendimientos mensuales del IPc. La técnica que emplearemos será el desarrollo de una regresión por cada empresa, tomando como variable dependiente los rendimientos de las acciones y como variable independiente el cambio porcentual del IPc. La estimación de los parámetros será a partir de mínimos cuadrados ordinarios (MCO).

A continuación se plantea el modelo:

$$
R_{j, t}=\alpha+B_{j} R_{m t}+\varepsilon_{t}
$$

donde: $\alpha=$ constante de regresión; $R_{j, t}=\frac{P_{j, t}-P_{j, t-1}}{P_{j, t-1}}$ con $P_{j, t}$ igual al precio mensual de la acción:

$$
j ; R_{m t}=\frac{I P C_{t}-I P C_{t-1}}{I P C_{t-1}}
$$

que representa el cambio porcentual del IPC y $\varepsilon_{t}=$ término de error.

Se llevó a cabo la prueba de raíces unitarias para contrastar el carácter no estacionario de las series a fin de verificar rigurosamente nuestras conjeturas sobre la media no constante en los datos. Para ello, se considera el trabajo de Dickey y Fuller (1979), y obtuvimos que los datos no son estacionarios. Por ello, recurrimos al trabajo de Osborne (1959), el cual propone utilizar los cambios del logaritmo del precio para representar un proceso con incrementos independientes e idénticamente distribuidos. Por ello, consideramos que $R_{j, t} \approx \operatorname{Ln}\left(P_{j, t}\right)-\operatorname{Ln}\left(P_{j, t-1}\right)$ y

\footnotetext{
${ }^{5}$ Se considerarán los precios ajustados a dividendos y ampliaciones de capital.
} 
$R_{m, t} \approx \operatorname{Ln}\left(I P C_{j, t}\right)-\operatorname{Ln}\left(I P C_{j, t-1}\right)$, donde: $\operatorname{Ln}$ representa el logaritmo neperiano. Volvimos a contrastar la prueba de raíces unitarias considerando los cambios logarítmicos en las series de precios y del IPc y obtuvimos que los datos ya no contaban con tendencia, por lo que podemos trabajar con ellos y calcular la beta mediante el CAPM tradicional.

Los datos muestran que las betas son significativas y con valores en promedio en 0.96; esto no representa una característica de las economías emergentes, pero en nuestro caso fue un hallazgo totalmente diferente del que pensábamos que íbamos a tener, por lo cual rechazamos la primera hipótesis.

\section{Cálculo de la prima por riesgo}

Vamos a considerar la metodología de Fernández (2001, p. 411) que define el promedio esperado del mercado como: "el promedio de rendimientos realizables en el mercado en exceso de los rendimientos de bonos libres de riesgo a largo plazo".

Para la tasa libre de riesgo $\left(R_{f, t}\right)$ tomamos los Cetes a 28 días reflejando una tasa promedio de 2000 a 2007 de $0.7 \%$. Mientras que para el rendimiento esperado del mercado $E\left(R_{m, t}\right)$ consideramos el promedio del rendimiento mensual de 2000 a 2007 obteniendo en promedio una tasa de $2 \%$. Para obtener la prima del mercado calculamos la diferencia entre el rendimiento del mercado y la tasa libre de riesgo, esto es $\left(E\left(R_{m, t}\right)-R_{f, t}\right)$. La prima por riesgo es de $1.23 \%$ para el periodo considerado.

Para calcular el costo de capital tomamos como referencia la ecuación 2; es importante señalar que el costo de capital es una tasa nominal; para obtener la tasa real utilizamos la expresión $r=t n-\pi$, donde $r=$ tasa real, $t n=$ tasa nominal y $\pi=$ inflación observada en promedio durante el periodo de estudio.

Se presentan los promedios por industria del costo del capital anual real; podemos observar que el sector con mayor costo de capital es el de "comunicaciones y transportes", con una tasa de $24 \%$ de costo de capital, y la más baja la obtuvo el "sector servicios", con un costo de capital de 12\%. En promedio se tiene un costo de $19 \%$. Si hacemos un análisis más detallado tenemos que bajo el CAPM las tres empresas con mayor costo de capital son Elektra (28\%), TVAzteca (28\%) y GFAMSA (26\%), y las tres empresas con menor costo de capital son GAP (2\%), Kimber (9\%) у омA $(10 \%)$. 
CuAdro 1. Costo de capital promedio por industria de las empresas del IPC (mayo de 2007) (CAPM) ${ }^{6}$

\begin{tabular}{|l|c|}
\hline \multicolumn{2}{|c|}{$\begin{array}{c}\text { Promedio del costo de capital por industria para mayo de 2007 } \\
\text { (Porcentaje) }\end{array}$} \\
\hline Comercio & 22 \\
\hline Comunicaciones y transportes & 24 \\
\hline Construcción & 19 \\
\hline Extractiva & 17 \\
\hline Servicios & 12 \\
\hline Transformación & 13 \\
\hline Varios & 21 \\
\hline
\end{tabular}

\section{Cálculo del costo de Capital utilizando el CaPM-M $\left(\boldsymbol{\beta}_{d}\right)$}

El CAPM-M considera el riesgo de obtener rendimientos negativos y, por ello, en un mercado emergente como el mexicano ayuda a incrementar las betas y hacerlas estadísticamente más significativas. Para calcular la $\beta_{d}$ utilizamos el modelo de regresión presentado en la expresión (3). ${ }^{7}$ Obtuvimos en promedio una beta de 0.86, sorprendentemente más baja y contra lo encontrado en Estrada (2000 y 2001), por lo que rechazamos la hipótesis 1 que nos dice "Las betas calculadas a partir del CAPM tradicional son más bajas que las obtenidas mediante la metodología del CAPM-M”. Este hallazgo lo podemos atribuir a que el mercado de renta variable mexicano ha tenido evoluciones muy favorables en el precio de las acciones durante el periodo analizado.

Pero si comparamos las $R^{2}$ ajustadas tenemos que mejoran con el CAPM-M en 23 empresas y empeoran en 12 empresas, esto es $65 \%$ de las veces se refleja una mejora mediante la utilización del CAPM-M. Por lo que aceptamos la hipótesis 2 que nos señala que "la utilización del CAPM-M para calcular la beta modificada aumenta la emulación del modelo mediante un incremento de la $R^{2}$ ajustada en promedio".

Para calcular la tasa libre de riesgo y la prima por riesgo consideramos la misma información que utiliza el CAPM tradicional ya que, como se analizó ante-

\footnotetext{
${ }^{6}$ Para un análisis más detallado por empresa ver el anexo 2.

${ }^{7}$ Considerando las diferencias logarítmicas en el precio de las acciones y en el IPc.
} 
riormente, la diferencia entre el CAPM tradicional y el CAPM modificado recae en el cálculo de la beta.

Los resultados del costo de capital mediante CAPM-M se muestran en el cuadro 2. En promedio se tiene un costo de capital de 17\%. Mientras que los sectores con mayores tasas de rendimientos para los accionistas son el de "comunicaciones y transportes" y el de "varios", con una tasa de $22 \%$, por otro lado el costo de capital más bajo lo tiene el sector de "servicios" (con 10\%). En relación con los costos de capital por empresa tenemos que utilizando el CAPM-M las tres empresas con mayor costo de capital son TVAzteca (27\%), Alfa (27\%) y AMX $(24 \%)$ mientras que las tres empresas con menor costo de capital son GAP $(-1 \%)$, GFAMSA $(5.3 \%)$ y OMA $(7 \%)$.

CUADRO 2. Costo de capital promedio por industria

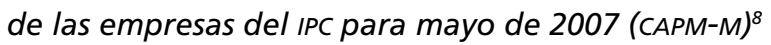

\begin{tabular}{|l|c|}
\hline \multicolumn{2}{|c|}{$\begin{array}{c}\text { Promedio del costo de capital (modificado) por industria para mayo de 2007 } \\
\text { (Porcentaje) }\end{array}$} \\
\hline Comercio & 18 \\
\hline Comunicaciones y transportes & 22 \\
\hline Contrucción & 17 \\
\hline Extractiva & 19 \\
\hline Servicios & 10 \\
\hline Transformación & 13 \\
\hline Varios & 22 \\
\hline
\end{tabular}

\section{RESUltados DE LAS ESTIMACIONES}

No podemos conocer el verdadero valor de $\beta$ pero podemos analizar cómo se comporta la $\beta$ al aumentar el tamaño de la muestra. En la gráfica 1 se presenta la evolución de los estimadores con el CAPM tradicional, y en la gráfica 2 la de las $\beta_{d}$ con el modelo CAPM-M (versión modificada). En los dos casos las betas llegan a estabilizarse al aumentar el tamaño de muestra, por lo que comprobamos que estos estimadores son consistentes en los dos modelos.

\footnotetext{
${ }^{8}$ Para un análisis más detallado por empresa véase el anexo 1.
} 
GrÁFICA 1. Estabilidad de $\hat{\beta}$ al aumentar el tamaño de n (modelo CAPM)

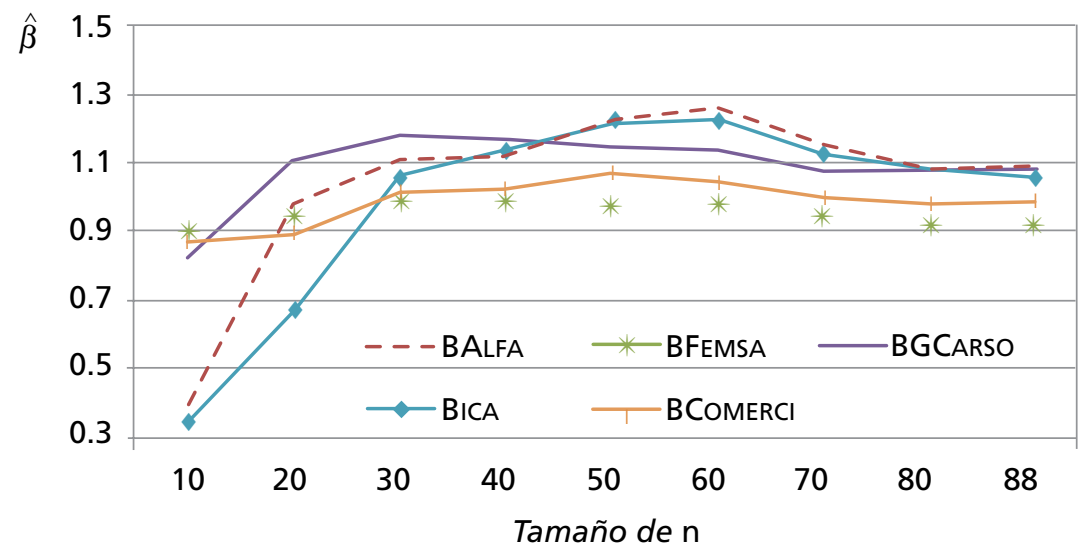

GráfICA 2. Estabilidad de $\hat{\beta}$ al aumentar el tamaño de $\mathrm{n}$ (modelo CAPM-M)

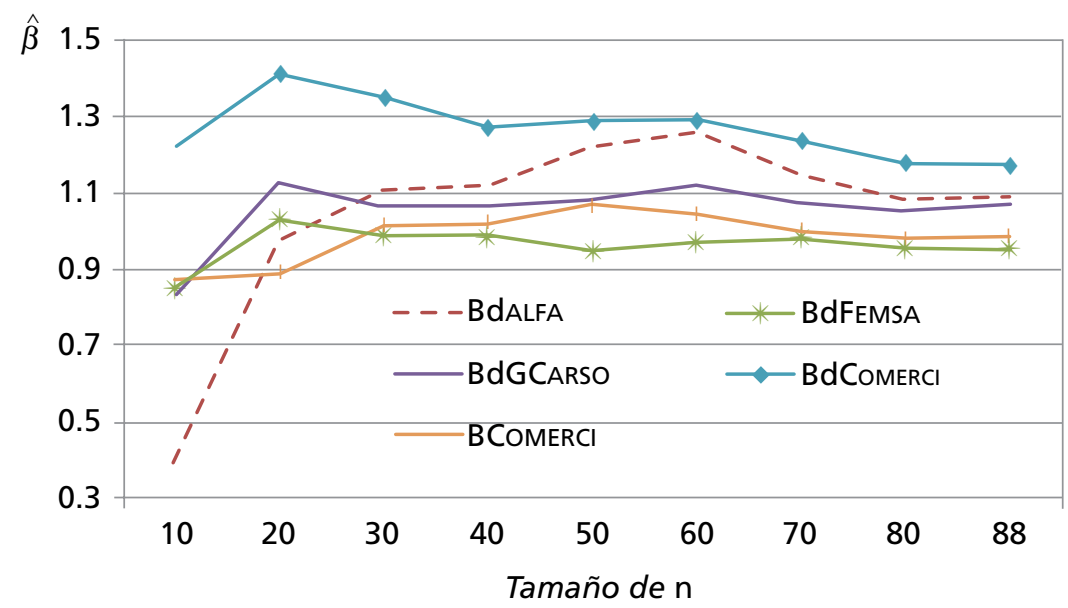

Para conocer la eficiencia del modelo optamos por encontrar los errores residuales de la regresión en los dos modelos, esto es:

$$
\sum_{i=1}^{n}(y-\hat{y})^{2} .
$$

En la gráfica 3 se presentan los hallazgos de los dos modelos, en donde encontramos que el modelo con menor error es el CAPM-M, por lo que es más eficiente. 
GráfICA 3. Suma de errores al cuadrado de los residuos en los dos modelos (CAPM у CAPM-M)

Suma de errores residuales

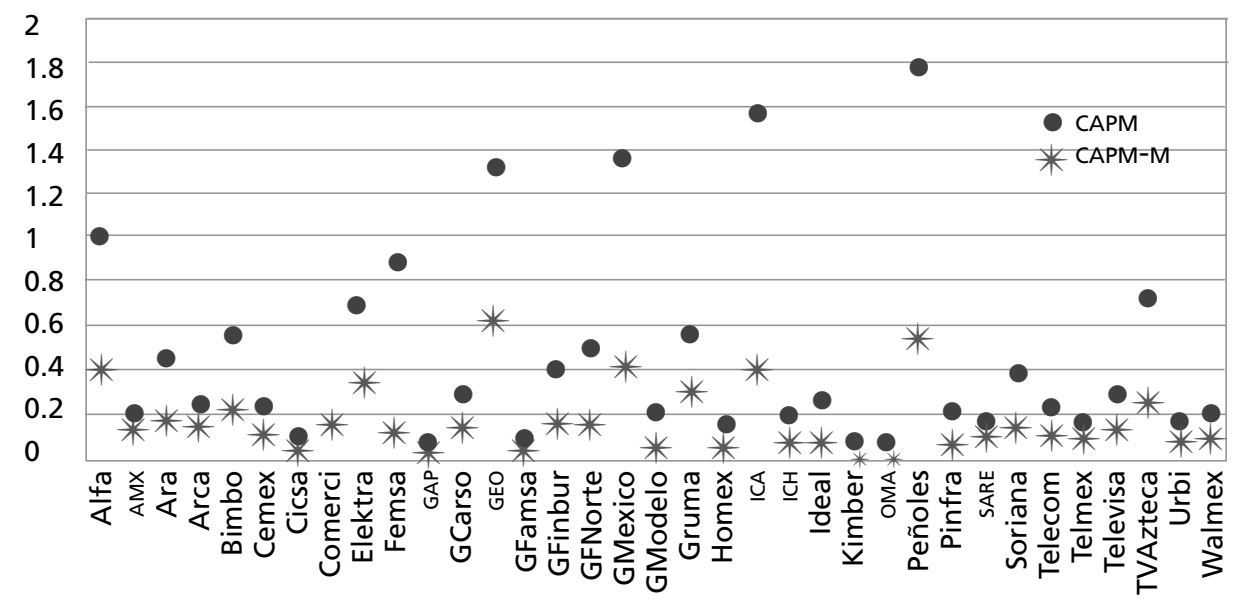

\section{Consideraciones en México}

Un problema de aplicación de los modelos CAPM y CAPM-M consiste en que la mayoría de las empresas en México son privadas, lo cual significa que no están inscritas en la Bolsa Mexicana de Valores. Easley y O'Hara (2004) demuestran que las empresas que no son públicas reflejan mayores costos de capital que las públicas ya que la información, cuando es privada, incrementa el riesgo en los inversionistas al no estar informados. Otro estudio que mantiene la misma idea es el propuesto por Lambert et al. (2005), el cual ilustra que las empresas con mayor calidad de información (públicas) no solamente afectan la percepción de los inversionistas, sino también tienen efectos positivos en los flujos de caja futuros, lo cual hace que los inversores estén dispuestos a disminuir la prima por riesgo de empresas. En México podemos señalar intuitivamente que las empresas privadas tienen un mayor riesgo de mercado ya que los inversionistas que apuestan en ellas no se encuentran diversificados, por lo que el costo de capital de éstas es mayor.

Una última consideración para México es que aplicamos el CAPM y CAPM-M para mostrar qué modelo era más conveniente utilizando las acciones que componen el IPC en México. Sin embargo, sabemos que estos modelos se pueden aplicar en agregados de actividades económicas como comercio, comunicaciones y transportes, construcción, etcétera. 
Aunque el análisis variaría, ya que en lugar de utilizar el rendimiento de la empresa como variable dependiente utilizaríamos el rendimiento de un grupo de empresas representativas del sector o bien el índice de un sector específico mientras que la variable independiente sería el IPC.

\section{Conclusiones}

En un mercado de capitales como el mexicano se cuenta con información imperfecta, por lo cual es importante que antes de aplicar cualquier modelo financiero se hagan los ajustes necesarios para su estudio. En primer lugar, calculamos el costo de capital según el supuesto de competencia perfecta utilizando el capital asset price model (CAPM) propuesto por W. Sharpe (1964), y lo comparamos con una metodología en donde no suponemos mercados imperfectos, hipótesis formulada y adaptada por J. Estrada (2000 y 2001) denominada capital asset price model modificated (CAPM-M). En contra de lo esperado, las betas calculadas a partir del CAPM tradicional son más altas que las obtenidas mediante la metodología del CAPM-M en las empresas que constituyen el índice de precios y cotizaciones (IPC). Encontramos que la beta bajo el enfoque tradicional es de 0.96 mientras que bajo el enfoque modificado es de 0.86 , lo cual significa que cualquier cambio en el rendimiento del mercado ocasionará cambios en la misma proporción en el rendimiento de la acción, mientras que bajo el enfoque tradicional los cambios en el rendimiento del mercado afectan $86 \%$ los rendimientos de las acciones.

La utilización del CAPM-M para calcular la beta modificada aumenta la emulación del modelo a partir de un incremento de la $R^{2}$ ajustada de $32 \%$ en promedio en el enfoque tradicional a $36 \%$ en el modificado en promedio. Los costos de capital en promedio presentaron valores de $17 \%$ y $19 \%$, en el enfoque tradicional y modificado, respectivamente, mientras que las desviaciones estándar oscilaron $6 \%$ en los dos enfoques.

Encontramos que en las dos metodologías las betas llegan a estabilizarse al aumentar el tamaño de muestra, por lo que comprobamos que estos estimadores son consistentes. Sin embargo, un resultado a favor del CAPM-M es que los errores residuales de la regresión son menores que en el CAPM, por lo que concluimos que el CAPM modificado es más eficiente.

Los sectores con mayores costos de capital fueron los de comunicaciones y transportes, comercio y varios, y los que obtuvieron menores costos son los de servicios y transformación. En estudios futuros se contempla la aplicación de este análisis a un mayor número de empresas para poder observar qué otros determi- 
nantes pueden influir en los costos de capital, como por ejemplo la periodicidad de la beta, el nivel de volatilidad de la beta, el nivel de bursatilidad de los precios de las acciones, así como el cálculo de costos de capital por agregados económicos como comercio, comunicaciones y transportes, construcción, etcétera.

Anexo 1

\begin{tabular}{|c|c|c|}
\hline Acciones & Sin constante & Valor crítico con $5 \%$ \\
\hline Alfa & -3.56 & -1.94 \\
\hline AMX & -3.70 & -1.94 \\
\hline Ara & -5.19 & -1.94 \\
\hline Arca & -4.31 & -2.60 \\
\hline Bimbo & -4.69 & -1.94 \\
\hline Cemex & -5.19 & -1.94 \\
\hline Cicsa & -2.78 & -1.96 \\
\hline Comerci & -5.30 & -1.94 \\
\hline Elektra & -4.60 & -1.94 \\
\hline Femsa & -5.18 & -1.94 \\
\hline GAP & -2.05 & -1.97 \\
\hline GCarso & -4.74 & -1.94 \\
\hline GEO & -4.33 & -1.94 \\
\hline GFamsa & -1.34 & $-1.98^{*}$ \\
\hline GFinbur & -6.13 & -1.94 \\
\hline GFNorte & -4.40 & -1.94 \\
\hline GMexico & -4.07 & -1.94 \\
\hline GModelo & -5.62 & -1.94 \\
\hline Gruma & -4.22 & -1.94 \\
\hline Homex & -3.86 & -1.95 \\
\hline ICA & -5.16 & -1.94 \\
\hline $\mathrm{ICH}$ & -3.36 & -1.95 \\
\hline Ideal & -2.07 & -1.96 \\
\hline Kimber & -2.30 & -1.95 \\
\hline OMA & ND & n.d.* \\
\hline Peñoles & -4.37 & -1.94 \\
\hline Pinfra & -1.78 & $-1.97^{*}$ \\
\hline SARE & -4.31 & -1.95 \\
\hline Soriana & -4.74 & -1.94 \\
\hline Telecom & -4.74 & -1.94 \\
\hline Telmex & -5.00 & -1.94 \\
\hline Televisa & -5.37 & -1.94 \\
\hline TVAzteca & -4.83 & -1.94 \\
\hline Urbi & -3.11 & -1.95 \\
\hline Walmex & -5.05 & -1.94 \\
\hline
\end{tabular}

* No son estacionarias en media; existencia de raíces unitarias. 


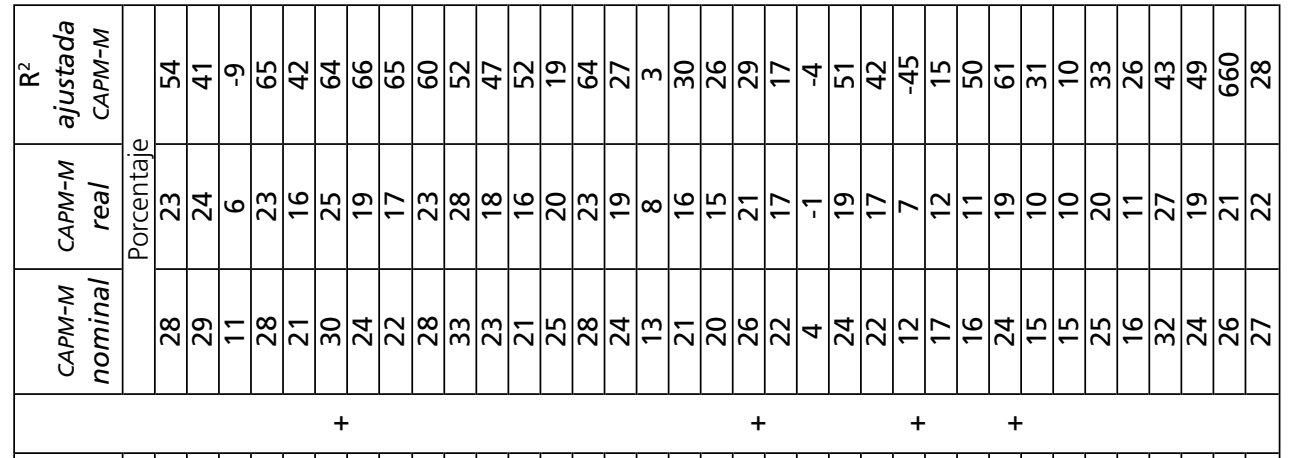

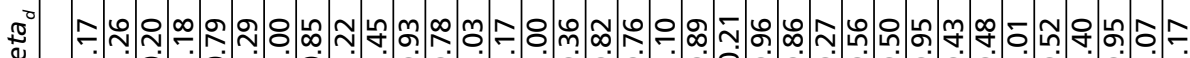

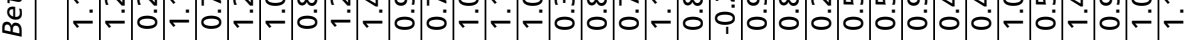

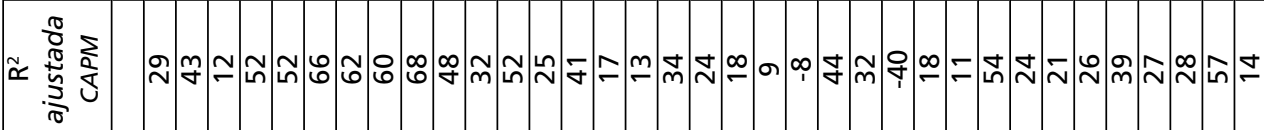

交

:๐

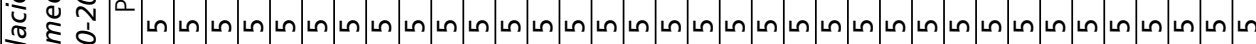
इ

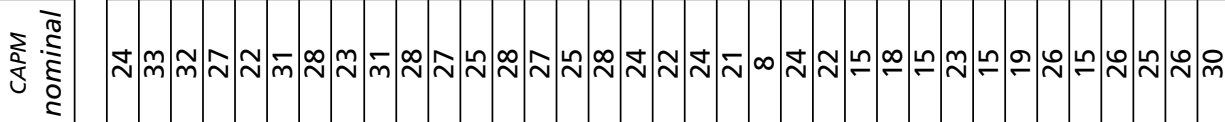

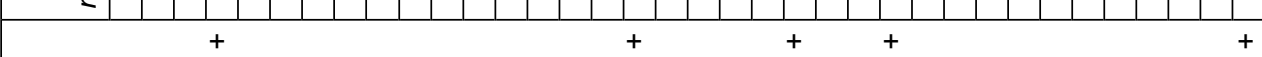

‡

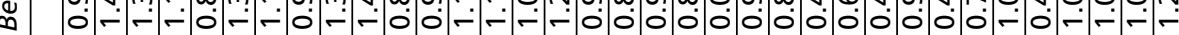
(1) :

ᄒ̀ $\quad$ (

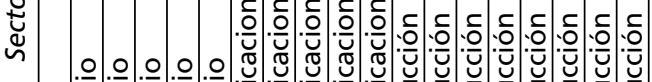

는ㄷำ 으.의.의 으 으

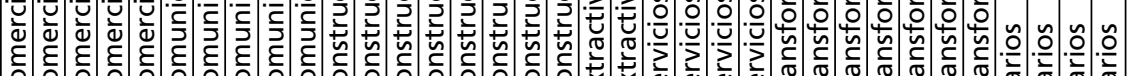
نे

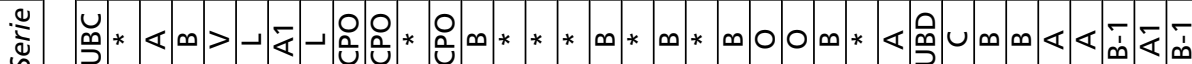




\section{REFERENCIAS RIBLIOGRÁFICAS}

Bawa, Vijays S., y Eric B. Lindenberg (1977), "Capital market equilibrium in a meanlower partial momennt framework", Journal of Financial Economics, vol. 5, núm. 2, pp. 189-200.

Dickey, David, y W. Fuller (1979), "Distribution of the estimators for autoregressive time series", Journal of the American Statistical Association, vol. 24, núm. 366, pp. 427-431.

Easley, D., y M. O'Hara (2004), "Information and the Cost of Capital", Journal of Finance, vol. 55, agosto, pp. 1553-1583.

Erb, Claude, Campbell Harvey, y Tadas Viskanta (1996), "Political risk, economic risk, and financial risk", Financial Analysts Journal, vol. 52, núm. 6, pp. 29-46.

Estrada, Javier (2000), "The cost of equity in emerging markets: A downside risk approach”, Emerging Markets Quarterly, vol. 4, núm. 3, pp. 19-30. (2001), "The cost of equity in emerging markets: A downside risk approach (II)", Emerging Markets Quarterly, vol. 5, núm. 1, pp. 63-72.

(2002), "Mean-Semivariance Behavior: An Alternative Behavioral Model", documento de discusión, IESE Business School.

Fama, Eugene F., y Kenneth R. French (1992), "The cross-section of expected returns", Journal of Finance, vol. 47, núm. 2, págs. 427-465.

Fernández, Pablo (2001), Valoración de empresas, Ediciones Gestión 2000, Barcelona, segunda edición revisada y ampliada.

Godfrey, Stephen, y Ramón Espinosa (1996), “A practical approach to calculating costs of equity for investment in emerging markets", The Bank of America Journal of Applied Corporate Finance, vol. 9, núm 3, pp. 80-89.

Harlow, Van, y Rao Ramesh (1989), "Asset pricing in a generalized meanlower partial moment framework: Theory and evidence", Journal of Financial and Quantitative Analysis, vol. 24, núm. 3, pp. 285-311.

Harvey, Campbell (1995), "Predictable risk and reurns in emerging markets", Review of Financial Studies, vol. 8, núm. 3, pp. 773-816.

Lambert, R., C. Leuz, y R. E. Verrecchia (2005), “Accounting Information, Disclosure, and the Cost of Capital", documento de discusión, University of Pennsylvania.

Markowitz, Harry M. (1959), Portfolio selection: Effcient diversification of investment. Yale University Press, New Haven.

Osborne, M. F. M. (1959), "Brownian motion in the stock market", en Cootner, Paul (comp.) (1964), The random chatacter of stock market price, MIT Press, Cambridge Massachussetts.

Sortino, Frank, y Robert van der Meer (1991), "Downside risk", The Journal of Portfolio Management, vol. 7, núm. 2, pp. 27-31. 